\title{
Etude de la protéolyse du fromage type "Manchego " au cours de l'affinage
}

\author{
par \\ M. RAMOS et I. MARTINEZ-CASTRO \\ Instituto de Productos Lacteos. C.S.I.C. Arganda del Rey, Madrid
}

\section{INTRODUCTION}

La maturation du fromage est un processus bien complexe, qui a lieu au travers de plusieurs changes biochimiques : fermentation du lactose, hydrolyse de la matière grasse, dégradation des protéines, etc. Ces phénomènes sont à l'origine de produits responsables du goût et de l'arôme caractéristiques, qui donnent au fromage ses propriétés organoleptiques particulières.

Malgré les très nombreuses études relatives à l'affinage des divers types de fromages, (Camembert [1], Cheddar [2, 3, 4, 5, 6 et 7], Montasio [8], Hervé [9], Vacherin [10], Gouda [11], Domiati [12], etc.) les références trouvées à l'égard du Manchego $[13,14,15]$ sont peu abondantes, bien qu'il soit le plus connu en Espagne.

Dans une étude de M. Roman [16] sur l'évolution de la flore microbienne du fromage Manchego, quelques caractéristiques chimiques (acidité, matière sèche, teneur en chlorures, lactose, azote total et soluble) ont été déterminées.

Edwards et Kosikowski [18] et Fox [19], à leur tour, ont signalé que l'électrophorèse, appliquée aux fractions protides, donne le jugement le plus convenable à ce sujet.

Dans le présent travail, afin d'obtenir une information au cours de la protéolyse au long de la maturation du fromage type Manchego, les changements subis par les fractions protides (caséines) sont évalués par techniques électrophorétiques, et l'évolution des matières azotées est dosé simultanément déterminant l'azote soluble et l'azote non caséinique.

Quant à la matière grasse, les indices de Reichert-Meissl, de Polenske, de Kirschner, et de réfraction, sont déterminés dans le but de vérifier si le processus d'hydrolyse est capable de les faire varier au long de l'affinage, puisque ces valeurs sont fixées dans la législa- 
tion espagnole pour la matière grasse du lait, provenant ou non de fromages affinés. La variation de ces indices a été déjà étudiée dans les divers types de fromages espagnols [17], au long de sa vie commerciale, mais pas dès son origine.

\section{MATERIELS ET METHODES}

\section{Préparation et prélèvement des échantillons}

Bien que le fromage Manchego soit, dès son origine, un produit du lait de brebis, aujourd'hui on peut trouver dans le marché des fromages "type Manchego "fabriqués avec des mélanges lait de vache - lait de brebis, et mème seulement avec du lait de vache. C'est pour cela que nous avons préparé six groupes de sept fromages avec du lait de brebis, l'un d'eux pur, les autres mélangés avec 10, 20, 40 et 100 p. 100 de lait de vache. Le sixième groupe est pur brebis, inoculé d'une souche de "starter », cultivé sur du lait de brebis.

Les fromages ont été préparés avec du lait pasteurisé, suivant la technique habituelle dans l'industrie : on ajoute comme "starter » une souche de $S$. lactis et $S$. cremoris (CHR Hansen's Laboratonium, Copenhague) cultivée sur du lait de vache, on laisse agir la présure (activité 1:150 000) pendant $40 \mathrm{mn}$ à $32^{\circ} \mathrm{C}$; puis le caillé est découpé, réchauffé à $32^{\circ} \mathrm{C}$ pendant $30 \mathrm{mn}$, pressé et salé dans un bain de saumure pendant $24 \mathrm{~h}$. L'affinage a eu lieu à $12^{\circ} \mathrm{C}$ pendant 10 mois environ. Tous les fromages, qui avaient un poids de $2,5 \mathrm{~kg}$ environ, ont développé le goût et l'arôme typiques des bons fromages Manchego. Seulement ceux fabriqués avec du lait de vache pur eurent des caractères organoleptiques légèrement différents.

\section{Méthodes d'analyse}

Les prélèvements ont été effectués sur ces six groupes de fromages, le premier dans le caillé, les suivants à $3,10,20,30,60,120$ et $180 \mathrm{j}$ d'affinage, prélevant un fromage entier de chaque groupe.

Les fromages prélevés sont découpés tout de suite par secteurs circulaires, et, puis la croûte enlevée, la pâte est rapée, mélangée soigneusement et conservée dans des flacons fermés à $2^{\circ} \mathrm{C}-4^{\circ} \mathrm{C}$. Les analyses suivantes sont effectuées :

L'acidité est déterminée par titration [20] et la matière sèche par gravimétrie [21]. L'azote soluble est dosé par solubilisation dans de l'eau chaude [22]. L'azote non caséinique est déterminé selon Sozzi et Shephard [10] : l'échantillon est dispersé dans de l'eau, puis précipité à $\mathrm{pH}$ 4,6 avec le tampon acide acétique - acétate de sodium et centrifugé. L'azote est dosé dans le surnageant par la méthode de Kjeldahl. L'azote total est dosé aussi par la méthode de Kjeldahl [23]. 
Les échantillons pour l'électrophorèse sont préparés selon la méthode de Pierre [24]. La quantité d'échantillon est adaptée à notre cas particulier : 3,6 g de fromage, rapés de l'intérieur de l'échantillon, sont broyés avec $30 \mathrm{ml}$ d'eau distillée dans un mix ; la graisse surnageante est éliminée, et le $\mathrm{pH}$ amené à 4,6 dans un tube de centrifugeuse. Puis le tube est centrifugé à 3000 r.p.m. pendant $5 \mathrm{mn}$, le surnageant est rejeté et le résidu dispersé dans $5 \mathrm{ml}$ d'urée $7 \mathrm{M}$. La graisse qui reste est maintenant éliminée avec $\mathrm{Cl}_{*} \mathrm{C}$. La fraction protide, dissoute dans l'urée $7 \mathrm{M}$, est ainsi prête pour l'électrophorèse. On a choisi comme support l'acétate de cellulose, par sa rapidité et commodité. La solution $(2 \mu \mathrm{l}$ environ) est déposée sur des rectangles de Cellagram (Shandon Scientific Co. Ltd., London) de $15 \times 8 \mathrm{~cm}$, au moyen d'un applicateur de sérum (Gelman Instrument Co., Ann Arbor, Michigan). On utilise un tampon véronal-véronal sodique à $\mathrm{pH} 8,6$, contenant de l'urée $5 \mathrm{M}$. Les conditions de l'électrophorèse sont : appareil pour l'électrophorèse horizontale, mod. Kohn U 77 avec stabilisateur Vokam (Shandon

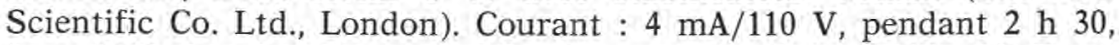
à $+4^{\circ} \mathrm{C}$. Les bandes sont colorées avec du Noir Amido $10 \mathrm{~B}$, et lavées avec de l'acide acétique à 5 p. 100 ; puis les rectangles sont séchés et traités avec ciclohexanone-éthanol (22:78) pour les rendre transparents. Les bandes sont mesurées par densitométrie avec un appareil mod. Cromoscan MK-II (Joyce, Lobl and Co. Ltd., Gateshead). La mobilité des bandes est rapportée à celle de la caséine $\beta$.

Les bandes principales (caséines $\alpha$ et $\beta$ ) sont identifiées par comparaison avec celles des laits d'origine.

La matière grasse est extraite par broyage du fromage avec sulfate de sodium et oxyde diéthylique [25]. Le solvant est chassé sous vide à $40^{\circ} \mathrm{C}$. Sur la matière grasse ainsi obtenue, les indices des acides gras volatils solubles et insolubles [26, 27] et de réfraction [28] sont déterminés.

\section{RESULTATS}

Les résultats sont réunis dans trois groupes, et seulement la valeur moyenne de chaque groupe est représentée : le premier groupe est formé par les deux séries de fromages pur brebis, le deuxième avec les trois séries de mélanges brebis-vache, le troisième avec la série pur vache.

La figure 1 montre l'évolution de la teneur en matière sèche et en acidité des trois groupes de fromages.

L'augmentation de la matière sèche est lente dans les premiers jours, et devient plus marquée entre le $3^{e}$ et le $4^{e}$ mois ; puis elle reste relativement stable.

Les variations de l'acidité sont peu sensibles. On peut observer un certain parallélisme dans tous les fromages; ceux de vache montrent des valeurs nettement inférieures. 


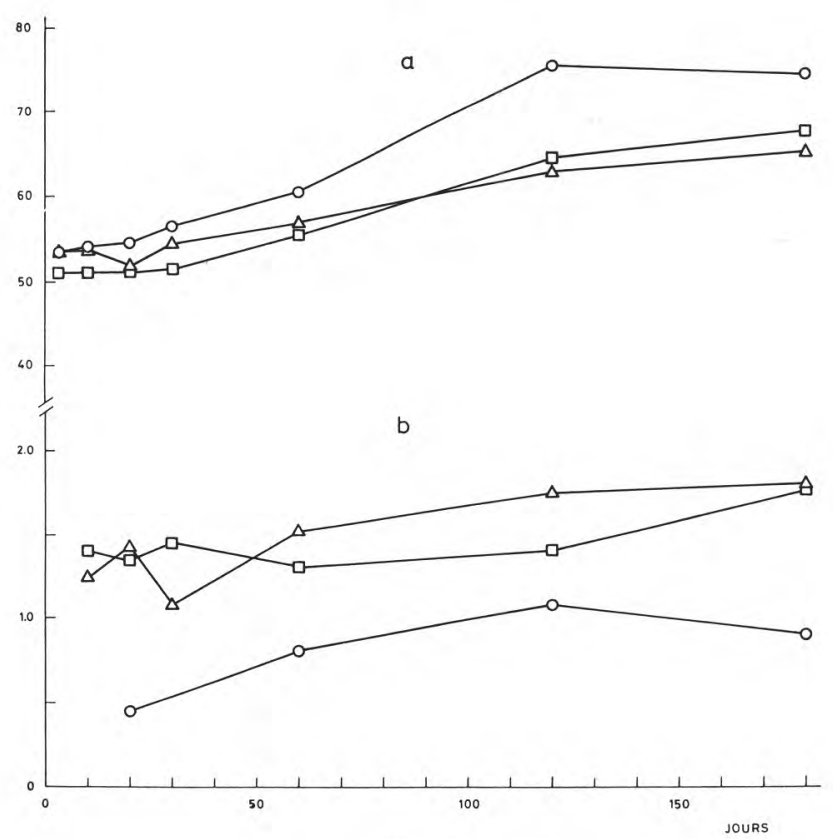

fig. 1

Evolution des teneurs en matière sèche : (a) et en acidité ;

(b) des trois groupes de fromages au long de l'affinage

( $\triangle$, pur brebis ; 0 , pur vache ; $\square$, mélanges).

Pour mettre en rapport les diverses fractions protides on a utilisé deux indices de maturation :

$$
\begin{aligned}
& \mathrm{IM}_{1}=\frac{\text { Azote soluble }}{\text { Azote total }} \times 100 \\
& \mathrm{IM}_{2}=\frac{\text { Azote non caséinique }}{\text { Azote total }} \times 100
\end{aligned}
$$

Les valeurs obtenues sont montrées dans la figure 2 .

On peut constater une augmentation très marquée de l'azote soluble $\left(\mathrm{IM}_{1}\right)$ vers le $20-30^{\mathrm{e}}$ jour, qui se poursuit jusqu'au $2^{\mathrm{e}}$ mois ; cette augmentation a lieu d'avance chez les fromages pur vache.

L'indice $\mathrm{IM}_{2}$ varie d'une façon très semblable, pourtant l'augmentation est plus graduelle.

On a examiné quelques fromages après 10 mois, et aucun de ces deux indices a montré à peine une variation par rapport aux valeurs qu'ils avaient au $6^{\mathrm{e}}$ mois. 

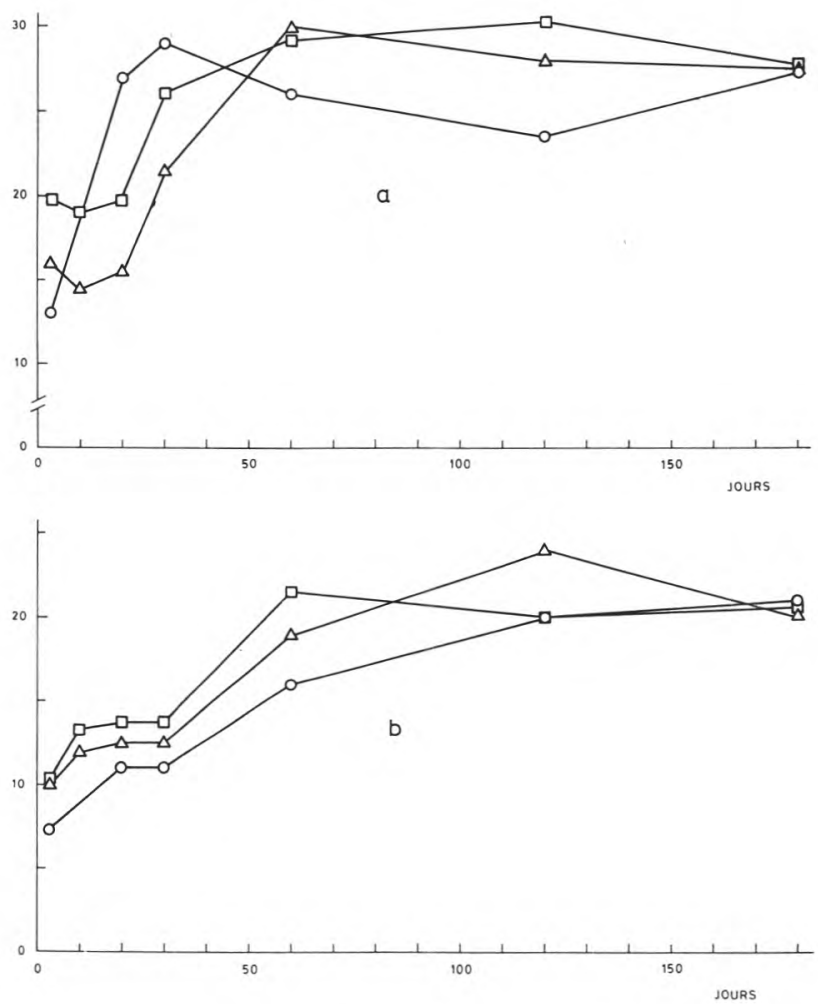

fig. 2

Variations des indices $\mathrm{IM}_{1}$ (a) et $\mathrm{IM}_{2}$ (b) des trois groupes de fromages au long de l'affinage $(\triangle$, pur brebis ; 0 , pur vache ; ․, mélanges).

Considérant ces résultats, il semble que le processus protéolytique principal a eu lieu dans les 2 premiers mois d'affinage ; mais l'électrophorèse des caséines va montrer que la dégradation est plus profonde, et que le cours de la protéolyse ne s'arrête pas à ce point.

Les figures 3, 4 et 5 montrent les diagrammes électrophorétiques des caséines des trois groupes de fromages. Ils sont bien différents, non seulement les uns des autres, mais aussi par rapport aux laits d'origine. Les résultats densitométriques sont exposés dans les tableaux 1 à 3 . Les composants sont numérotés selon leur mobilité, à partir de l'origine. Quelques bandes peu résolues, comme celles 2-3, 5-6 (dans les cas où elles sont présentes) sont intégrées ensemble. 
Les fromages de vache (fig. 3) montrent une bande (mobilité 2,5) qui apparaît déjà dans le caillé, et que nous appelons $\boldsymbol{\alpha}_{\mathrm{x}}$. Cette bande, produit de dégradation de la caséine $\alpha_{\mathrm{s}}$, augmente son intensité au long de l'affinage, jusqu'à devenir supérieure à celle de la caséine $\alpha_{\text {s }}$ même ; cela arrive vers le $20^{\mathrm{e}} \mathrm{j}$, quand l'indice $\mathrm{IM}_{1}$, a presque atteint sa valeur maximum. Néanmoins, le total des composants $3+4$ (caséine $\alpha_{s}+$ caséine dégradée $\alpha_{x}$ ) décroît, ce qui peut arriver comme résultat d'une protéolyse plus profonde, dont les produits finals sont difficilement détectables par l'électrophorèse.

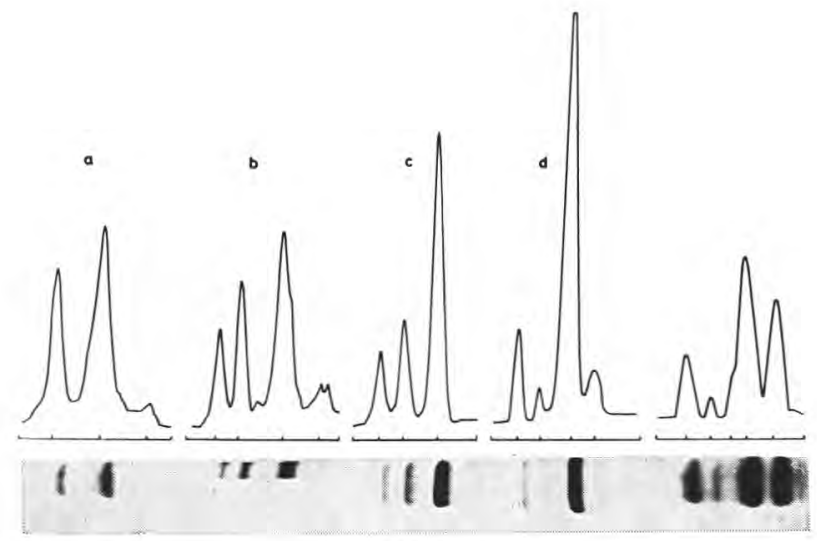

fig. 3

Phorégrammes et courbes densitométriques des caséines isolées des fromages de vache : (a) lait ; (b) caillé ; (c) fromage, $3 \mathrm{j}$; (d) id., $20 \mathrm{j}$. ; (e) id., $180 \mathrm{j}$.

Au-delà du $2^{\mathrm{e}}$ mois, les bandes de mobilité 0,33 augmentent d'intensité ; une autre bande faible (mobilité 1,18) est observée aussi à partir du $4^{\mathrm{e}}$ mois, ce qu'on peut attribuer à un commencement de dégradation de la caséine $\beta$.

Les phorégrammes du groupe pur brebis (fig. 4) sont plus complexes que ceux de vache. La caséine $\alpha_{\mathrm{s}}$ du lait est formée par deux bandes, et elle donne lieu, dans le caillé, à un autre groupe de deux-trois bandes, dont la mobilité est supérieure $(2,2,2,3)$.

Les pourcentages relatifs, exposés dans le tableau 2, ne montrent pas de variations sensibles. Il faut signaler qu'à mesure que le temps passe, le processus de dégradation continue (malgré les valeurs pas trop élevées des indices $\mathrm{IM}_{1}$ et $\mathrm{IM}_{2}$ ) car les bandes deviennent diffuses, ce qui rend très imprécise la mesure densitomiétrique. Ainsi, des fractions visibles au $2^{\mathrm{e}}$ mois ne peuvent pas être détectées, même visuellement, au $8^{\mathrm{e}}$ mois. Cela peut être dû à la formation de produits à poids moléculaire élevé. 


\section{$T A B L E A U 1$}

Composition de la fraction insoluble à $\mathrm{pH} 4,6$ des fromages de vache (pourcentages relatifs)

\begin{tabular}{c|r|r|r|r}
\hline \multirow{2}{*}{ Jours } & \multicolumn{4}{|c}{ Composants } \\
\cline { 2 - 4 } & 1 & $2(\beta)$ & $3\left(\alpha_{\mathrm{s}}\right)$ & $4\left(\alpha_{\mathrm{x}}\right)$ \\
\hline & & & & \\
3 & 3,1 & 62,1 & 22,9 & 11,9 \\
10 & 4,3 & 52,7 & 25,9 & 17,1 \\
20 & 4,7 & 60,4 & 15,9 & 19,0 \\
30 & 5,4 & 63,7 & 11,5 & 20,4 \\
60 & 23,5 & 51,7 & 12,3 & 13,5 \\
120 & 21,0 & 63,3 & 2,5 & 13,1 \\
180 & 33,2 & 53,9 & 1,8 & 13,1 \\
& & & & \\
\hline
\end{tabular}

TABLEAU 2

Composition de la fraction insoluble à $\mathrm{pH}$ 4,6 des fromages de brebis (pourcentages relatifs)

\begin{tabular}{c|c|c|c|c}
\hline \multirow{2}{*}{ Jours } & \multicolumn{4}{|c}{ Composants } \\
\cline { 2 - 4 } & 1 & $2(\beta)$ & $3\left(\alpha_{\mathrm{s}}\right)$ & $4\left(\alpha_{\mathrm{x}}\right)$ \\
\cline { 2 - 4 } 3 & & & & \\
10 & 7,9 & 62,5 & 19,6 & 10,0 \\
20 & 9,9 & 63,7 & 11,7 & 14,7 \\
30 & 6,7 & 67,6 & 13,8 & 11,9 \\
& 10,0 & 66,5 & 12,8 & 10,7 \\
\hline
\end{tabular}




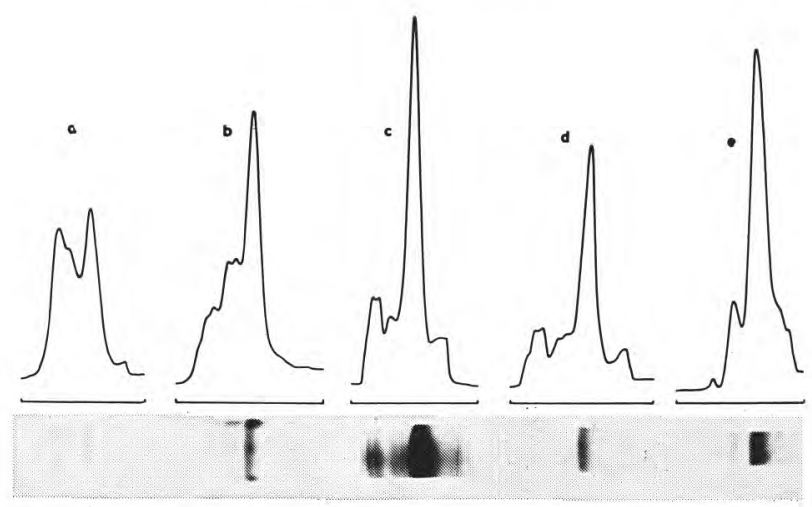

fig. 4

Phorégrammes et courbes densitométriques des caséines isolées des fromages de brebis : (a) lait ; (b) caillé ; (c) fromage, $10 \mathrm{j}$; (d) id., $20 \mathrm{j}$; (e) id., $180 \mathrm{j}$.

Il faut signaler aussi que nous n'avons pas observé de dégradation de la caséine $\beta$ dans aucun fromage de ce groupe.

Les phorégrammes des fromages mélangés sont encore plus complexes : à côté des bandes typiques du fromage de brebis, qu'on a déjà vu dans la série précédente, on peut distinguer une autre bande dont la mobilité $(2,4)$ correspond à celle du composant $\alpha_{x}$ du fromage de vache.

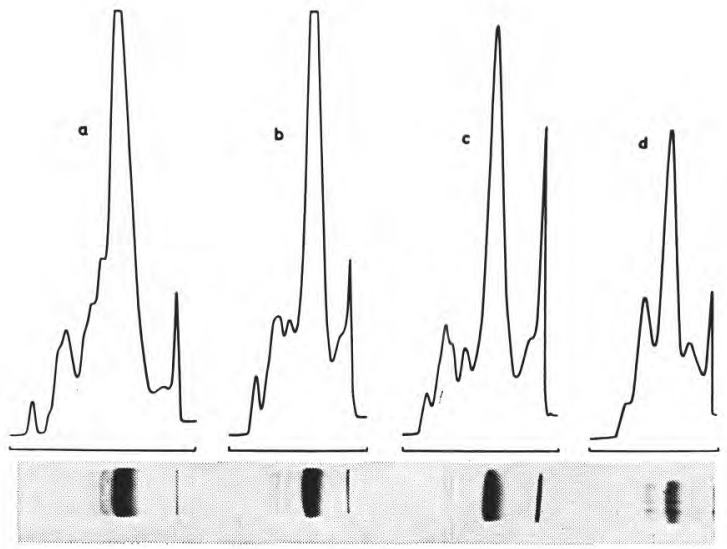

fig. 5

Phorégrammes et courbes densitométriques des caséines isolées des fromages mélangés brebis-vache : (a) $5 \mathrm{j}$; (b) $10 \mathrm{j}$; (c) $30 \mathrm{j}$; (d) $180 \mathrm{j}$. 
Les composants 1,4 et 5 apparaissent depuis l'emprésurage. Le $n^{\circ} 1$ montre une augmentation graduelle d'intensité, tandis que celle-ci diminue dans ceux $n^{\text {os }} 4$ et 5 , de façon semblable aux fromages pur vache.

\section{TABLEAU 3}

Composition de la fraction insoluble à $\mathrm{pH}$ 4,6 des fromages fabriqués avec mélange brebis-vache (pourcentages relatifs)

\begin{tabular}{|c|c|c|c|c|c|}
\hline \multirow{2}{*}{ Jours } & \multicolumn{5}{|c|}{ Composants } \\
\hline & 1 & $2(\beta)$ & $3 \alpha_{\mathrm{s}}$ & $\begin{array}{l}4\left(\alpha_{x}\right) \\
\text { ovine }\end{array}$ & $\begin{array}{l}5\left(\alpha_{x}\right) \\
\text { bovine }\end{array}$ \\
\hline 3 & 4,7 & 56,2 & 20,2 & 16,4 & 2,5 \\
\hline 10 & 7,8 & 66,0 & 9,8 & 14,5 & 1,9 \\
\hline 20 & 8,4 & 70,1 & 9,3 & 11,2 & 1,0 \\
\hline 30 & 9,6 & 69,3 & 12,4 & 7,9 & 0,8 \\
\hline 60 & 14,9 & 7,12 & 12,8 & 1,1 & - \\
\hline
\end{tabular}

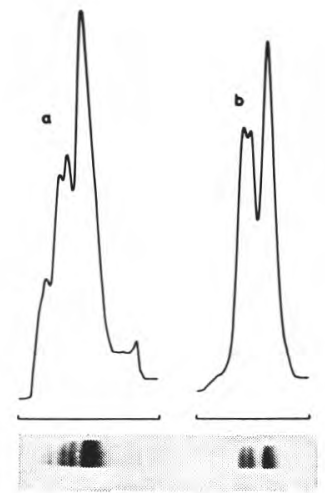

fig. 6

Phorégrammes et courbes densitométriques d'un fromage de brebis fabriqué avec (a) et sans "starter" (b) au $3^{\mathrm{e}} \mathrm{j}$ d'affinage.

Au-delà du $2^{\mathrm{e}}$ mois, les bandes deviennent diffuses.

La figure 6 montre le diagramme électrophorétique d'un fromage de brebis fabriqué sans "starter ". Les caséines ne sont pas altérées, car toutes les bandes sont égales à celles du lait de départ. C'est avec le temps, et quand l'affinage se poursuit, que des produits de dégradation commencent à apparaître. 
TABLEAU 4

Variation des indices de la matière grasse des fromages au long de l'affinage (valeurs initiale et finale)

\begin{tabular}{|c|c|c|c|c|c|c|c|c|c|}
\hline & & \multicolumn{2}{|c|}{ I. Reichert-Meissl } & \multicolumn{2}{|c|}{ I. Polenske } & \multicolumn{2}{|c|}{ I. Kirschner } & \multicolumn{2}{|c|}{ I. Réfraction } \\
\hline & & a & $\mathrm{b}$ & $\mathrm{a}$ & $\mathrm{b}$ & $\mathrm{a}$ & $\mathrm{b}$ & $\mathrm{a}$ & b \\
\hline \multirow{3}{*}{ Brebis } & 1 & 33,7 & 34,0 & 8,8 & 9,1 & 21,8 & 25,4 & 1,4546 & 1,4541 \\
\hline & 2 & 33,1 & 33,3 & 8,7 & 9,1 & 23,7 & 25,4 & 1,4542 & 1,4540 \\
\hline & 1 & 34,3 & 33,3 & 8,8 & 8,6 & 21,4 & 21,9 & 1,4543 & 1,4538 \\
\hline \multirow[t]{2}{*}{ Mélanges } & 2 & 33,4 & 33,6 & 7,5 & 7,8 & 22,9 & 24,9 & 1,4543 & 1,4540 \\
\hline & 3 & 32,9 & 33,0 & 6,9 & 7,5 & 21,9 & 25,2 & 1,4543 & 1,4541 \\
\hline Vache & 1 & 30,3 & 29,8 & 2,7 & 3,1 & 22,0 & 22,5 & 1,4546 & 1,4546 \\
\hline
\end{tabular}

a : Valeur initiale $\left(4^{\mathrm{e}} \mathrm{j}\right)$

b : Valeur finale ( $6^{\mathrm{e}}$ mois $)$. 
Le tableau 4 montre les valeurs des indices de matière grasse. On $n^{\prime} a$ indiqué que les valeurs initiales $\left(4^{e} \mathrm{j}\right)$ et finales ( $6^{\mathrm{e}}$ mois). Les chiffres varient d'une série à l'autre mais restent constants pendant l'affinage.

\section{DISCUSSION}

Malgré les nombreux travaux versant sur la protéolyse du fromage, il y a une grande diversité dans leurs conclusions, car les facteurs contribuant à chaque processus protéolytique $(\mathrm{pH}$, température, présure, etc.) sont divers aussi.

Nous avons observé que l'emploi des "starters " dans l'emprésurage joue un rôle très important dans la dégradation des caséines. Des fromages fabriqués avec de la présure sans "starter ", pasteurisés ou non, développent une protéolyse peu signalée, et leur diagramme électrophorétique est donc très semblable à celui du lait d'origine, au moins pendant le $1^{\text {er }}$ mois d'affinage. Nous supposons que ce phénomène est dû aux enzymes protéolytiques des microorganismes des "starters ". Actuellement, on travaille dans notre laboratoire à ce sujet.

D'un autre côté, le "starter " cultivé dans du lait de brebis a montré un comportement très similaine à celui cultivé dans du lait de vache. La protéolyse qu'ils provoquent ne nous a pas permis de trouver aucune différence entre eux.

Quant à la dégradation des caséines, c'est la $\alpha_{s}$ qui commence à se dégrader, suivie, en certains cas, par la $\beta$ [5]. Au contraire, Weckx et Vanderpoorten [9] trouvent que la caséine $\beta$ est dégradée chez le fromage Hervé dans la $3^{e}$ semaine, bien qu'ils utilisent des présures microbiennes, qui donnent lieu à d'autres types de dégradation.

Nous avons observé que le processus protéolytique semble différent quand le fromage type Manchego est fabriqué avec du lait de vache ou de brebis. On peut affirmer que la caséine $\alpha$ est dégradée depuis l'emprésurage, car il y a une bande (composant $n^{\circ} 4$ ) de mobilité supérieure à celle de la caséine $\alpha_{s}$, qui est déjà visible dans le caillé. Cette bande devient plus intense de même que la caséine $\alpha_{s}$, bien que tous les deux diminuent leur intensité dans les fromages très affinés.

La caséine $\beta$ est restée relativement stable. Aucune dégradation apparente est détectable dans les fromages de brebis examinés, même au $10^{\mathrm{e}}$ mois, tandis que dans ceux de vache la caséine $\beta$ semble commencer à se dégrader à partir du $4^{\mathrm{e}}$ mois.

Creamer [29] a trouvé, dans des fromages Cheddar et Gouda, âgés de 11 et 30 mois, quelques peptides avec un poids moléculaire 
élevé et une petite mobilité, qu'il considère comme produits de dégradation de la caséine $\beta$, ce qui pourrait avoir quelque relation avec la bande à mobilité 0,33 , que nous avons observé aussi.

Quant à l'hydrolyse de la matière grasse, elle est généralement très limitée dans les fromages à pâte dure. Les variations trouvées dans les indices d'acides volatils étant très petites, la perte de ces acides aura donc peu d'importance ; c'est-à-dire, si ces indices ne sont pas convenables pour l'étude de l'affinage, ils permettent, au contraire, de caractériser la matière grasse des fromages, tant des frais que des affinés.

\section{Remerciements}

Nous voulons remercier le docteur Yubero Cârcamo de l'Ecole des Industries Laitières (M. Agriculture) de Madrid, pour sa collaboration pendant la fabrication des fromages.

\section{R és u m é}

On a étudié la dégradation protéolytique de six séries de fromages type Manchego, fabriqués avec diverses proportions de lait de vache et de brebis. L'évolution des fractions azotées est déterminée, et la dégradation des caséines est suivie par l'électrophorèse sur l'acétate de cellulose. Dans les fromages additionnés de "starters " la dégradation de la caséine $\alpha_{s}$ est visible dès l'emprésurage, et poursuivie au long de l'affinage. La caséine $\beta$ reste inaltérée dans les fromages de brebis, tandis que dans ceux de vache, des bandes de dégradation sont observées à partir du $4^{\circ}$ mois. Quelques indices classiques de la matière grasse sont étudiés aussi.

\section{S u m m a r y}

The proteolytic degradation of sixth séries of Manchego cheese, made with different proportions of sheep's and cow's milk was investigated. The evolution of some nitrogen fractions is studied and the degradation of caseins is followed by cellulose acetate electrophoresis. The degradation of $\alpha_{\mathrm{s}}$ casein in cheeses made with starters begins in renneting and continues during ripening. $\beta$ casein remains unaltered in sheep's cheeses, but some degradation bands appeared on the fourth month in cow's cheeses. Some milk fat values of cheeses are also given. 


\section{Bibliographie}

[1] Lenoir (J.) (1963). - Le Lait, 423-424, 154.

[2] Marth (E. H.) (1963). - J. Dairy Sci., 46, 869.

[3] Mabiitt (L. A.) (1961). - J. Dairy Res., 28, 303.

[4] Harper (W. J.), Kristoffersen (T.) (1966). - J. Dairy Sci., 39, 1773.

[5] Ledford (R. A.), O'Sullivan (A. C.), Nath (K. R.) (1966). - J. Dairy Sci., 49, 9.

[6] Lindouist (B.), Storgards (T.) (1959). - Proc. Intern. Dairy Congr. London, 679.

[7] Richardson (B. C.), Creamer (K.) (1973). - N.Z. J. Dairy Sci. Tech., 8, 46.

[8] Corradini (C.), Batristotti (D.) (1973). - Sc. Tecn. Latt. Casear., 24, 11.

[9] Weckx (M.), Vanderpoorten (R.) (1973). - Milchwiss., 28, 332.

[10] Sozzi (J.), SHEPHERD (D.) (1972). - Le Lait, 514, 203.

[11] Vanderpoorten (R.), Weckx (M.) (1972). - Neth. Milk Dairy J., 26, 47.

[12] Abd El-Salam (M. H.), El-Shibiny (S.) (1973). - Austr. J. Dairy Technol., 28,61 .

[13] Gallego (M.) (1956), - Rev. Esp. Lecheria, 20, 71.

[14] Lopez Lorenzo (P.), SAnz Perez (B.), Burgos (J.) (1962). - Annales de Bromatologia, 14, 221.

[15] Wirotama (I. P. G.), Ney (K. M.), Freitag (W. G.) (1973). - Z. Lebensmitt.unters, 153, 78.

[16] Roman Pinana (M.) (1975). - Le Lait, 547, 401.

[17] Villanua (L.), Carballido (A.) (1969). - Anal. Bromatol., 21, 241.

[18] Edwards (J. L.), Kosikowski (F. V.) (1969). — J. Dairy Sci., 52, 1675.

[19] Fox (P. F.) (1969). - J. Dairy Sci., 52, 1214.

[20] A.O.A.C. Official Methods (1970) $\mathrm{n}^{\circ} 16021$.

[21] Norme Internationale F.I.L.-I.D.F., 4:1958.

[22] Norme Internationale F.I.L.-I.D.F., 25:1964.

[23] Casares (R.) (1967). - Tratado de Análisis Quimico. Tomo III. Ed. Casares, Madrid.

[24] Pierre (A.), Portmann (A.) (1970). - Ann. Technol. Agric., 19, 107.

[25] Norme Internationale F.I.L.-I.D.F., 32:1965.

[26] Norme Internationale F.I.L.-I.D.F., 37:1966.

[27] A.O.C.S. Official Methods Cd-5-40.

[28] Norme Internationale F.I.L.-I.D.F. 7A:1969.

[29] Creamer (L. K.) (1975). - J. Dairy Sci., 58, 287. 